Ebisu

Études japonaises

Études japonaises

58 | 2021

2011-2021 : crises, ruptures et nouvelles dynamiques.

Dix ans après la triple catastrophe du 11 mars

\title{
Reconstruire son monde par les mots. Rêves et lettres dans les enquêtes du séminaire Kanebishi sur la catastrophe du 11 mars
}

Anne Gonon

\section{OpenEdition}

Journals

Édition électronique

URL : https://journals.openedition.org/ebisu/6102

DOI : 10.4000/ebisu.6102

ISSN : 2189-1893

Éditeur

Institut français de recherche sur le Japon à la Maison franco-japonaise (UMIFRE 19 MEAE-CNRS)

Édition imprimée

Date de publication : 1 novembre 2021

Pagination : 285-296

ISSN : $1340-3656$

Référence électronique

Anne Gonon, «Reconstruire son monde par les mots. Rêves et lettres dans les enquêtes du séminaire Kanebishi sur la catastrophe du 11 mars », Ebisu [En ligne], 58 | 2021, mis en ligne le 01 novembre 2021, consulté le 13 avril 2022. URL : http://journals.openedition.org/ebisu/6102 ; DOI : https:// doi.org/10.4000/ebisu.6102 


\title{
Reconstruire son monde par les mots Rêves et lettres dans les enquêtes du séminaire Kanebishi sur la catastrophe du 11 mars
}

\author{
Anne Gonon*
}

Toute catastrophe, industrielle, nucléaire ou naturelle, s'accompagne de la mort. Celle du 11 mars 2011 ne fait pas exception, mais si les médias ont régulièrement comptabilisé le nombre de victimes, aucune image de ces mêmes victimes n'a circulé dans les journaux ou à la télévision.

Cependant, derrière cette comptabilité froide mais qui nous indique l'ampleur de la catastrophe en pertes de vie humaine et animale, se profilent des personnes ayant eu une famille, ayant vécu une vie plus ou moins longue, des êtres humains avec un visage, une histoire. C'est cette disparition tragique d'une vie que le monde moderne ne veut plus affronter, cachant ces corps morts que les dessins de Georges Ferdinand Bigot (18601927), lors d'un tsunami survenu au début du $x^{e}$ siècle dans la même région de Fukushima, nous montraient. Et pourtant, la réalité est la même - certains ont survécu, d'autres ont disparu, emportés par le tsunami, et se pose toujours la question du deuil.

Ce texte est une note de lecture de Watashi no yume made, ai ni kite kureta: 311 nakihito to no sore kara 私の夢まで、会いに来てくれた一3・11 亡き人とのそれから (Tu es venu à ma rencontre, jusqu'au bout de mon rêve : vivre avec les disparus du 11 mars), Tokyo, Asahi shinbun shuppan 朝日新聞出版, 2018, et de 3.11 Reisei ni dakarete: tamashi to inochi no ikasarekata 3.11霊性に抱かれて一魂といのちの生かされ方 (Être embrassée par les âmes : comment faire vivre âme et vie), Tokyo, Shin.yōsha, 2018.

* Professeure à l'université Dōshisha. 
Certes, les médias évoquent régulièrement les cérémonies commémoratives organisées par les municipalités, ou la région, pour rappeler la triple catastrophe et profiter de cette occasion pour réactiver les mesures de sécurité en organisant des exercices collectifs. Mais que dire du travail de deuil personnel, de ce rapport essentiel aux morts qui doit s'accomplir?

Une équipe d'étudiants appartenant au séminaire d'un professeur d'une université du Tōhoku s'est attelée à la tâche de comprendre les différentes façons dont les survivants à la triple catastrophe faisaient le travail de deuil. Le résultat est publié en plusieurs volumes.

À la différence de la plupart des recherches de bonne qualité conduites sur la vie des victimes de la catastrophe qui s'intéressent aux stratégies de résilience, ou de résistance par la mise en place de structures collectives pour protester ou s'organiser ensemble pour trouver des moyens alternatifs de continuer à vivre là où a eu lieu la catastrophe, les travaux de cette équipe évoquent la vie ordinaire et quotidienne. Et dans cette situation, parler de la vie, c'est aussi parler de la mort, c'est-à-dire de l'épaisseur de la vie humaine et de sa fragilité.

Cette attention des chercheurs est au cœur de leur projet, porter " un regard juste et bienveillant dirigé sur une réalité individuelle", comme le disait Iris Murdoch, voire plus encore, une réalité intime ${ }^{1}$.

\section{Un grand projet : donner la parole aux habitants des zones dévastées}

L'initiateur du projet est un sociologue du nom de Kanebishi Kiyoshi 金菱清 qui enseigne alors à l'université Tōhoku Gakuin². Il a décidé de faire travailler ses étudiants, pour la plupart originaires de la région dévastée, sur la mémoire de cet événement. L’ouvrage intitulé 3.11 shōtotsu no kiroku: 71nin ga taiken shita otsunami/genpatsulkyodai jishin 3.11 衝突の記録一 71 人が体験した大津波・原発・巨大地震 (Notes du choc du 11 mars 2011 : tsunami, accident nucléaire, grand tremblement de terre tels que les ont vécus 71 personnes), paraît en 2012 et est la première exploration des voix

1. Iris Murdoch, The Sovereignty of Good, Routledge, 2013, p. 37 (version digitale).

2. Il est désormais professeur de sociologie à l'université Kwansei Gakuin. 
des victimes de cette triple catastrophe. Conscient de la différence existant entre les expériences selon qu'on a vécu le tsunami, le tremblement de terre ou l'accident nucléaire, le professeur Kanebishi classe ces témoignages selon les trois types de désastre. Le projet va se transformer avec le temps mais l'exploration de la parole va se poursuivre, avec l'idée que l'écriture peut être une thérapie. Une nouvelle impulsion est donnée avec une enquête demandée à des étudiants et publiée en 2016 sous le titre de Yobisamasareru reisei no shinsaigaku 呼び覚まされる霊性の震災学 (Désastrologie des âmes qu’on a rappelées). Le chapitre 1 , intitulé «La ville que les morts fréquentent : le fantôme du chauffeur de taxi ", évoque l'expérience étonnante que fait un chauffeur de taxi, environ trois mois après le tremblement de terre ${ }^{3}$. Il prend en charge une femme qui, malgré la chaleur de l'été, est vêtue d'un manteau. Elle veut se rendre dans un lieu qui surprend le chauffeur, car ce lieu a été complètement dévasté. Plus encore quand il lui demande si elle a froid, elle répond d'une voix tremblante : "Suis-je morte? » Le chauffeur, surpris, regarde alors dans le rétroviseur, et ne voit personne sur le siège arrière.

À la suite de la parution de l'ouvrage, des médias locaux ont mis en avant certains des cas présentés, notamment cette rencontre entre un chauffeur de taxi et un fantôme, déclenchant un afflux de témoignages dans lesquels les victimes parlent de leurs sentiments, de ce rapport avec les âmes des personnes décédées ou portées disparues. Le professeur décide alors de concentrer son attention sur tous ces témoignages, ces voix qui veulent être entendues, et il lance, en 2016, un projet sur le thème de la "mémoire du désastre " qui doit traiter de la relation avec la mort, ou plus exactement le phénomène des âmes défuntes sur les lieux des désastres. Ce rapport est, selon lui, au centre de ce que doit être une science du désastre telle qu'il la développe dans son ouvrage ultérieur de la même année (2016) : Shinsaigaku nyümon: shiseikan kara no shakai kōso 震災学入門一死生観から の社会構想 (Introduction à la désastrologie : penser le social à partir du sentiment de la vie et de la mort [c’est-à-dire de la logique de la vulnérabilité]).

Le séminaire a buté sur de nombreux obstacles: faire travailler des jeunes sur le thème de la mort dans des zones qu'ils connaissaient parfois

3. Kanebishi Kiyoshi Séminaire (dir.), "Yobisamasareru reisei no shinsaigaku : 3.11 sei to shi no hazama de» 呼び覚まされる霊性の震災学一 $3 \cdot 11$ 生と死のはざまで, Tokyo, Shin.yōsha 新曜社, 2016, p. 4-5. 
intimement, trouver l'énergie afin de mener une enquête alors qu'on est soi-même bouleversé par la situation, poser directement la question de la mort requiert une grande délicatesse pour être acceptée par les interlocuteurs. Kanebishi Kyoshi a compris qu'il était confronté à un phénomène total qui demandait une approche ethnographique. La recherche a été lente et les entretiens menés par les étudiants du séminaire ont d'abord eu lieu dans des espaces publics tels que les mairies et les temples.

De ces travaux de terrain, ont été tirées plusieurs publications ${ }^{4}$ :

- Hiai : ano hi no anata e tegami o tsudzuru 悲愛一あの日のあなたへ手紙をつ

づる (Amour dans la tristesse : t'écrire depuis ce jour, Tokyo, Shin.yōsha, 2017.

- 3.11 Reisei ni dakarete : tamashi to inochi no ikasarekata 3.11霊性に抱か れて一魂といのちの生かされ方 (Être embrassée par les âmes : comment faire vivre âme et vie), Tokyo, Shin.yōsha, 2018.

- Watashi no yume made, ai ni kite kureta: 311 nakibito to no sore kara 私の 夢まで、会いに来てくれた一3・11 亡き人とのそれから (Tu es venu à ma rencontre, jusqu'au bout de mon rêve : vivre avec les disparus du 11 mars), Tokyo, Asahi shinbun shuppan 朝日新聞出版, 2018.

- Eiketsu : ano hi no watashi e tegami o tsudzuru 永訣一あの日のわたしへ手紙 をつづる (Séparation éternelle : les lettres que tu m’as adressées depuis ce jour), Tokyo, Shin.yōsha, 2021.

Les titres des ouvrages indiquent clairement la position scientifique adoptée qui est l'exploration des liens que tissent les survivants à travers les rêves et l'écriture avec leurs proches disparus.

\section{Recréer une vie à partir des liens entre vivants et morts}

Il n'est pas aisé de présenter l'ensemble de ces ouvrages qui tentent délicatement de nous donner accès à la fois à la douleur ressentie devant la perte (ou la disparition) et au travail de deuil qui tisse de nouveaux liens avec les proches disparus, au moyen de l'écriture et du rêve.

L'anthropologue Veena Das observe que « la mort est marquée par sa non narratibilité et sa rupture dans le langage : le langage ordinaire se transforme

4. Nous ne retenons que les ouvrages du séminaire produits après 2016. 
dans le processus de narrer la mort, spécialement quand la mort est survenue en violation des normes culturelles d'une bonne mort ${ }^{5}$ ". Dans la langue japonaise, comme en français, il existe une distinction claire entre parler et conter. Parler se dit hanasu 話す et conter kataru 語る ${ }^{6}$. Avec le jeu des caractères chinois qui permet de proposer une autre combinaison de syllabes identiques, le champ sémantique des mots s'amplifie. Hanash $i$ 話 peut s'écrire avec un caractère qui signifie " parole ", mais aussi avec un caractère qui signifie "libération ». Le verbe hanasu sera alors utilisé pour signifier que parler veut dire projeter vers l'extérieur sa propre expérience, s'en séparer pour créer un lieu commun d'échange avec l'autre. Kataru 語る signifie " conter ", mais également par le même processus de choisir d'autres caractères ayant la même prononciation kata 型: mettre en forme. Le conte ou le récit sera ainsi la " parole mise en forme ", selon des liens de causalité qui aboutissent à une conclusion; cette démarche, adoptée dans l'ouvrage de 2012, libère par la parole tout en créant de nouveaux liens avec les disparus.

$\mathrm{Au}$ sein des cultures modernes, le traitement des traumatismes est devenu une pratique courante, mais des formes alternatives sont reconnues également comme légitimes. Le contexte socioculturel japonais dispose, en matière de traitement des victimes, d'un arsenal de soins psychologiques pouvant ressembler à celui existant autour du traitement des traumatismes dans les pays occidentaux : certes, la notion de $\mathrm{PTSD}^{7}$ est connue, mais son développement est récent - elle a été pour la première fois évoquée en 1995 à la suite du tremblement de terre de Kobe - et l'approche est mise en œuvre principalement à la demande des autorités publiques. Depuis 1995, l'approche en PTSD est développée autour de la formation professionnelle et de la mise en place de structures d'accueil : la psychiatrie japonaise ne

5. Veena Das, "Language and Body. Transactions in the Construction of Pain ", in Kleinman Arthur, Das Veena, Lock Margaret M., Social Suffering, Berkeley, University of California Press, 1997, p. 97-93. Une « bonne mort » est une mort qui survient su terme d'une vie de longueur moyenne.

6. Je reprends ici la thèse de Chiba Kazumiki, Gendai bungaku wa "shinsai no kizu " o iyaseru ka : 3. 11 no shögeki to merankorī 現代文学は「震災の傷」を癒せるかー3.11の 衝撃とメランコリー (La littérature contemporaine peut-elle apaiser les «blessures du désastre »? Le choc du 3.11 et la mélancolie), Kyoto, Mineruva shobō ミネルヴァ書房, 2019, p. 16-25.

7. Trouble de stress post-traumatique. 
se pratique pas en ambulatoire, mais elle hospitalise. La destruction des hôpitaux lors du 11 mars 2011 a rendu la pratique psychiatrique difficile, difficulté redoublée par le manque de personnel médical.

À un niveau plus ordinaire, des lieux tels que les écoles ou les centres municipaux offrent une forme simple de soins dits "soins du cœur " kokoro no kea [care] ふのケア - qui laisse ainsi une place aux traitements alternatifs, issus des pratiques ordinaires, des symptômes post-catastrophe. Ces initiatives peuvent être individuelles ou collectives. Nous retrouvons dans ce processus ce que Perera observe dans son étude des esprits du Sri Lanka, en déclarant que "de tels efforts individuels réussissent parce qu'ils jouissent d'une large reconnaissance communautaire, d'une acceptation et d'une légitimation. Dans ce sens, ce sont les efforts de la communauté pour soigner et s'en sortir ${ }^{8}$."

Les recherches menées par le séminaire Kanebishi relèvent d'une tentative de saisir en quoi la catastrophe a ouvert la voie à une nouvelle façon d'être humain, en appréhendant la douloureuse réalité. Pascale Molinier parle d'angoisse morale, pour désigner ce sentiment de n'avoir plus de nom connu pour nommer ce qui se passe ${ }^{9}$. Pour vivre, il faut s'attacher aux capacités de création d'image (imaginariser, dit-elle) à partir desquelles un processus d'emprise sur la réalité sera possible. Paradoxalement, ne pourrait-on pas dire que le rêve produit cette "imaginarisation »?

\section{Rêver}

Prêter attention aux rêves, mais aussi reconnaître la place qu'ils occupent dans le travail de deuil est un des grands mérites de ces recherches, alors que la sociologie a longtemps été incapable de l'intégrer dans la mise en forme du monde ${ }^{10}$. Peut-être peut-on lire dans cette ouverture au surnaturel une

8. Sasanka Perera, «Spirit Possessions and Avenging Ghosts: Stories of Supernatural Activity Narrative of Terror and Mechanisms of Coping and Remembering ", in Veena Das et al. (dir.), Remaking a World - Violence, Social Suffering and Recovery, Berkeley, University of California Press, 2001, p.183.

9. Pascale Molinier, Le care monde. Trois essais de psychologie sociale, Paris, ENS Éditions, 2018 (version électronique).

10. Le travail récent de Bernard Lahire constitue dans ce domaine une belle nouveauté. Bernard Lahire, L'Interprétation sociologique des rêves, Paris, La découverte, 2018. 
particularité de la culture japonaise. Le pavillon du rêve du temple Hōryūji de Nara est connu pour exprimer l'importance du rêve dans la culture des anciens Japonais. Cette culture du rêve a nourri non seulement les individus, mais également la littérature, le théâtre et la religion. Notamment les pièces du théâtre nô en témoignent dans lesquelles un homme assoupi (le plus souvent un bonze) va rêver et être visité par un " esprit " mort de mort violente, masqué. Celui-ci va engager un dialogue avec le bonze et conter son histoire afin de trouver l'apaisement. Les paroles kotoba 言葉 sont entremêlées de mélodie pour exprimer les sentiments, et les émotions des deux protagonistes.

\section{Des pratiques locales}

Le corps individuel n' est pas cette " topie impitoyable ", " ce lieu sans recours où je suis condamné » dont parle Michel Foucault ${ }^{11}$. Suivant la conception du monde bouddhique, l'individu est toujours appréhendé dans son environnement familial, social et naturel. Dans la région du Tōhoku, qui reste un lieu très religieux où se mêlent le shintō et le bouddhisme, les pratiques chamaniques sont restées très vivaces : on trouve des conteurs, appelés kataribe 語り部 qui sont chargés de converser avec les disparus, mais aussi des femmes chamanes appelés itako イタコ ou ogamisama オガミサマ selon les régions, capables de mettre en relation les morts et les vivants. Leur nombre a considérablement diminué, mais il en subsiste dans la région du Tōhoku, où elles pratiquent des consultations individuelles ou en groupe. Elles sont chargées de mener des rituels d'apaisement de l'âme des morts, mais il leur arrive aussi de transmettre les volontés des morts aux vivants. En raison des destructions des lieux de vie qui ont provoqué la dispersion de la communauté et la distanciation des affiliations, le recours aux chamanes a été moins pratiqué, mais des cas ont été recensés dans des bourgs touchés par le tsunami tels que Takeda (département d'Iwate).

11. Michel Foucault, Le Corps utopique suivi de Les Hétérotopies, Fécamp, Nouvelles Éditions Lignes, 2019. 


\section{La voix des morts}

La frontière entre le monde réel et le monde de l'au-delà est poreuse, ainsi que nous le présente le dessin animé Le Voyage de Chibiro de Miyazaki $\mathrm{Hayao}^{12}$, où les esprits rôdent, cachés mais toujours présents et prêts à se manifester, ou Les contes de Tōno de Yanagita Kunio écrits en 1903, dont le $99^{\mathrm{e}}$, intitulé Le Tsunami, évoque la rencontre que fait un homme avec sa femme morte. C'est surtout cette forme qu'a prise la communication entre les deux mondes ${ }^{13}$.

La pratique bouddhique de posséder un autel dans sa maison s'accompagne du dépôt d'offrandes classiques telles un bol de riz, mais de façon plus populaire, surtout quand il s'agit d'enfants, du dépôt de leur peluche, de leur boisson ou de leurs vêtements préférés. Mais la survenue du tsunami a compliqué la tâche de deuil, en dispersant les objets ou en les enfouissant sous les décombres. Ainsi, la recherche des objets ayant appartenu aux personnes disparues a formé les prémisses du travail de deuil; malgré la difficulté de la tâche, elle a semblé d'autant plus essentielle que les corps des personnes disparues n’ont pas été immédiatement retrouvés. Tout objet le plus ordinaire retrouvé était perçu par les vivants comme le premier signe de la réappropriation du lien avec leurs proches disparus. C'est ce que Veena Das appelle la « descente dans l'ordinaire » ou « mutuelle absorption du violent et de l'ordinaire " : un homme redécouvre un livre que lisait sa femme, un autre porte son attention sur les tasses utilisées quotidiennement avant le tsunami. Ainsi ces objets deviennent-ils des supports d'un dialogue qui s'engage avec les morts ou avec les disparus.

Le dialogue avec les personnes disparues prend aussi une forme plus intériorisée, celle du rêve.

Depuis la catastrophe, les témoignages d'esprits rôdant dans les rues dévastées par le tsunami ont ranimé les vieilles croyances religieuses. Et les morts parlent pour conter leurs regrets, assouvir leur colère.

L'enquête du séminaire Kanebishi a été menée en 2017, principalement dans le département de Miyagi, parfois jusqu'à Fukushima, auprès d'environ mille personnes. L'ouvrage sur les rêves, issu de cette enquête, paru en

12. Miyazaki Hayao 宮崎駿, Sen to Chihiro no kamikakushi 千と千尋の神隠し, 2001.

13. Yanagita Kunio 柳田國男, Töno monogatari 遠野物語 (Les légendes de Tōno), Tokyo, Shūeisha bunko 集英社文庫, 1991 . 
2018, est composé de quatre parties : la première partie s'intitule "rêve, vivre le présent ", la seconde "de petites âmes ", la troisième "frontière entre rêve et réalité » et la quatrième "penser le rêve ». Si l'on souhaite rendre justice au travail d'entretien qui a été fait par les étudiants, grâce auquel apparaît la singularité de chaque rencontre avec la mort et les conditions de surgissement d'un signe des disparus, tout résumé est impossible. Nous allons donc présenter un ou deux cas, choisis de façon arbitraire.

Mais ce qu'expriment les rêves que font les survivants, ce sont souvent des paroles d'apaisement. Les rêves semblent être le lieu du care, le temps où les morts viennent encourager les vivants à continuer de vivre, en assurant que la mort les réunira quand le temps sera venu. Certains survivants voient en rêve leurs enfants grandir, d'autres rester identiques à ce qu'ils étaient à l'âge de leur mort. Dans le cas de cette triple catastrophe, la violence de la mort est visible en ce qu'elle a coupé la vie d'enfants qui, dit un des pères, "n'ont pas pu vivre la durée d'une vie qu'on estime normale».

Mais nous ne sommes pas en présence d'esprits violents malgré la mort violente vécue.

\section{L'ambiguïté des rêves}

Le premier récit "Dieu m'a donné juste un peu de temps" est celui de Suzuki Yumiko, une mère qui a perdu son fils Hidekazu, âgé alors de douze ans, emporté par le tsunami (p. 33). Dans les premiers temps de sa disparition, elle a utilisé son téléphone portable pour noter les rêves dans lesquels son fils a commencé d'apparaître, un ou deux mois après le tsunami : son fils lui parlait, par exemple en demandant " pourquoi n'es-tu pas là? Tu n'es pas là, alors je pleure et je ne bouge pas ". Dans ses rêves, la mère ne voit qu'un petit enfant de trois ans, ce qui lui évoque la mort de tous les enfants emportés par le tsunami.

Toutefois, peu à peu, les rêves se sont espacés, devenant de moins en moins perturbants sans que le souvenir de son fils s'efface pour autant. Elle a arrêté de transcrire ses rêves. De nouveau, un an après la catastrophe, elle rêve de son fils - elle le voit de loin, parmi ses camarades, revêtu de l'uniforme des collégiens. Cela se passe une semaine avant la cérémonie de remise des diplômes de l'école primaire. Madame Ide pense qu'il est venu la revoir, car il a disparu avant d'entrer au collège. 
Son fils apparaît également dans les rêves des autres membres de la famille, toujours dans une scène courte. Avec le temps, le sentiment de madame Suzuki a changé et, si elle n'oublie pas que la mort peut surgir à tout moment, elle a organisé la vie de sa famille en n'oubliant pas les anniversaires et les habitudes de son fils disparu. L'effacement des rêves dans lesquels il apparaît est désormais pour elle un soulagement, car le réveil où elle reprend conscience de la disparition de son fils est une douleur. Elle déclare ainsi : « je sens que Hideokazu est à mes côtés, et c'est beaucoup mieux comme ça $\mathrm{a}^{14}$. »

\section{Dialogue dans le rêve}

Ce récit appartient à la deuxième partie et il est fait par une grand-mère qui a perdu son petit-fils (p. 119). Elle se reproche de ne pas être allée le chercher à la sortie de l'école. Il lui apparait dans ses rêves souvent après avoir prié devant l'autel bouddhique ou, de façon plus inattendue, lorsqu'elle pleure ou quand elle s'adresse à lui ; il peut être triste, facétieux, boudeur; il se comporte comme l'enfant qu'il était, réclamant des étrennes, mais dans les rêves il apparaît généralement joyeux. Les rêves sont pour sa grand-mère le moyen de vérifier qu'il n'est pas seul dans l'au-delà. Une fois il lui est apparu plus âgé, il avait grandi.

\section{Écrire}

La stupeur ressentie face à l'ampleur de ce qu'on appelle désormais la « catastrophe de Fukushima " a provoqué une aphasie dans le monde des lettres. Des auteurs comme le prix Nobel Ōe Kenzaburō 大江健三郎, ont ensuite expliqué les raisons de cette impossibilité d'écrire. Puis, peu à peu, dans un mouvement contraire, la parole est apparue comme nécessaire : des textes courts, des romans de fiction ( $\mathrm{La}$ Centrale en chaleur, Takahashi Gen.ichirō ${ }^{-15}$ ), des poèmes ont été écrits, surtout depuis 2016. Mais l'écriture peut être un geste spontané quand on n'est pas professionnel. Les lettres écrites par les habitants des zones dévastées sont un autre des médias utilisés par les chercheurs du séminaire

14. Tu es venu à ma rencontre, jusqu'au bout de mon rêve, p. 38 .

15. Takahashi Gen.ichirō 高橋源一郎, Koisuru genpatsu 恋する原発, Tokyo, Kōdansha 講談社, 2011. Traduction en français : La Centrale en chaleur, par Cardonnel Sylvain, 2013, Paris, Books Edition. 
Kanebishi pour aider à l'expression de la parole dans un souci thérapeutique, et saisir ainsi ce travail de rencontre entre ceux qui ont perdu des proches et les personnes extérieures. Afin d'aider à l'expression de l'expérience de cette triple catastrophe, en 2012 ces chercheurs ont sollicité des témoignages peu de temps après la catastrophe. Mais dans l'ouvrage paru en 2017, c'est tout autre chose qui est tenté : les membres de l'équipe du professeur Kanebishi ont accordé une place centrale à d'autres pratiques telles que les adresses aux disparus, les lettres rédigées au fil des années. Pourquoi choisir la forme des lettres?

À la différence des paroles prononcées lors des entretiens, paroles qui sont pesées, organisées, contées en vue du rapport avec l'interlocuteur, qui prend quasiment figure d'enquêteur, les mots confiés aux lettres sont différents. Ainsi « ces mots qu'on adresse aux personnes les plus chères, ne sont-ils pas des actes qui rappellent fortement les liens rompus par le désastre? Par les mots que chacun confie à une lettre, la réalité du désastre acquiert pour la première fois une réalité qui s'inscrit profondément dans la vie quotidienne ${ }^{16}$. "

Un terme a été choisi, forgé pour exprimer le sentiment qui transparaît dans ce style épistolaire et qui donne son titre à l'un des ouvrages de l'enquête. S’il existe le terme Hiai transcrit 悲哀, la transcription 悲愛 n'existe pas. Le critique littéraire Wakamatsu Eisuke 若松英輔 l'a forgé pour exprimer la complexité des sentiments des habitants de la région : « le sentiment qui semble fou de revoir la personne aimée disparue ou morte est enfoui au cœur d'un silence gonflé de tristesse ${ }^{17}$ ".

Les lettres sont publiées sans aucun commentaire et leurs auteurs sont présentés en fin d'ouvrage : lettre d'une mère à sa fille alors âgée de six ans, emportée par le tsunami alors qu'elle était dans le bus scolaire, lettre d'un homme exilé loin de son village contaminé par la radioactivité qui déplore la perte de son chien et de son pays natal, etc.

Une des premières lettres de l'ouvrage exprime le doute moral, à la fois au sein de l'écriture et comme un doute existentiel. Elle s'intitule "Lettre qui ne parviendra pas à son destinataire ${ }^{18}{ }^{2}$. En voici le début :

16. Kanebishi Kiyoshi Séminaire, Amour dans la tristesse, 2017, p. XII.

17. Ouvrage à paraître de Wakamatsu Eisuke, Tamashī ni fureru : daishinsai to, ikiteiru shi 魂にふれる 大震災と、生きている死 (Effleurer les âmes, Le grand tremblement de terre et la mort en vie).

18. «Todokanai tegami» 届かない手紙, p. 34. 
Hé, maman,

Quand les gens meurent, qu'est-ce qu'ils deviennent?

$\mathrm{Si}$ on meurt, tout est fini; avec le temps qui passe, la chair change de couleur et commence à pourrir,

On est incinéré, on est réduit en os, on finit par n’être rien ("zéro »).

Voilà ce que j'ai pensé, moi qui, dans le séisme du Japon de l'Est, ai découvert les corps de mon père et de ma mère.

Et alors sans glace ni eau, j’ai préparé leurs corps, je les ai veillés, incinérés.

J'ai compris ce que devenaient les corps mais

L'âme, le cœur, où cela va-t-il?

\section{Conclusion}

Parmi les nombreux ouvrages ont tenté de comprendre les effets de la triple catastrophe sur les habitants des départements touchés, le projet du professeur Kanebishi présenté ci-dessus occupe une place particulière. D’une part, ce projet s'est étalé sur les dix ans qui ont suivi la catastrophe et son évolution offre ainsi la possibilité de comprendre le processus de transformation des individus touchés dans leur vie quotidienne, mais également de saisir comment le contact avec ces individus a nourri une recherche attentive à rendre compte de ce qui se passait et de forger de nouveaux outils pour y parvenir. D'autre part, outre le souci pédagogique essentiel qu'il a mis en place en proposant aux étudiants de son séminaire de mener ce travail d'enquête, Kanebishi Kiyoshi a recueilli des témoignages inestimables. Ceux-ci rendent compte que si une politique de prise en compte des populations a besoin de proposer des moyens de traitement des traumatismes que des psychologues professionnels peuvent assurer, il existe d'autres moyens que les populations elles-mêmes sont à même de mettre en place. Ces voix/voies alternatives sont plus fortes que les classiques mesures de « soin du cœur» (kokoro no kea 心のケア) qui ont essaimé après la catastrophe. On peut ainsi comprendre le projet scientifique du professeur Kanebishi de créer une science des désastres, une "désastrologie " qui s'appuie sur la parole des victimes dans laquelle le rapport aux morts est essentiel pour reconstruire une vie individuelle et communautaire. 